\title{
A produção do conhecimento em Distúrbios da Comunicação: análise de periódicos (2000-2005)
}

\section{Knowledge production in Speech-Language Pathology and Audiology: analysis of peer-reviewed journals (2000-2005)}

\author{
Ana Paula Berberian'1, Léslie Piccolotto Ferreira² ${ }^{2}$ Lilian Cássia Bornia Jacob Corteletti ${ }^{3}$, \\ Juliana Bueno Meirellles de Azevedo ${ }^{4}$, Jair Mendes Marques ${ }^{5}$
}

\begin{abstract}
RESUMO
Objetivo: Analisar parte da produção de conhecimento da área dos Distúrbios da Comunicação. Métodos: Análise dos artigos publicados nos periódicos: Revista de Distúrbios da Comunicação; Revista da Sociedade Brasileira de Fonoaudiologia; Revista Fonoatual e Revista Pró-fono, no período de 2000 a 2005. Variáveis examinadas: temática, faixa etária do público alvo, tipo de intervenção e contexto institucional. Resultados: Foram publicados 724 artigos. A temática mais pesquisada, de forma isolada ou combinada, foi Linguagem Oral (282), seguida de Audiologia (232), Motricidade Oral (91) e Voz (62). A faixa etária mais estudada foi de três a dez anos (145). A atuação de diagnosticar e/ou avaliar foi a mais relatada (547), em contexto clínico (542), escolar (63) e hospitalar (32). Conclusão: Esses números refletem o aumento das publicações desses periódicos. Há necessidade de uma distribuição mais equilibrada das temáticas, faixas etárias, tipos de intervenções e contextos institucionais nas produções da área. Novos estudos em torno da produção do conhecimento devem ser realizados visando o registro histórico do que tem sido pesquisado e a definição de ações e campos estratégicos para o implemento da pesquisa na área.
\end{abstract}

Descritores: Publicações periódicas; Publicações científicas e técnicas; Estatística \& dados numéricos; Pesquisa; Ciência

\section{INTRODUÇÃO}

A ampliação da produção do conhecimento no Brasil tem levado diferentes áreas a realizar um balanço dos estudos e pesquisas que vem sendo desenvolvidas, por considerarem a importância de tal análise no processo de construção da memória

Trabalho realizado na Universidade Tuiuti do Paraná - Curitiba (PR), Brasil, e na Pontifícia Universidade Católica de São Paulo - PUCSP - São Paulo (SP), Brasil.

(1) Doutora, Professora do Curso de Fonoaudiologia e do Programa de Estudos Pós Graduados em Distúrbios da Comunicação da Universidade Tuiuti do Paraná - UTP - Curitiba (PR), Brasil.

(2) Doutora, Professora Titular da Faculdade de Fonoaudiologia e do Programa de Estudos Pós-Graduados em Fonoaudiologia da Pontifícia Universidade Católica de São Paulo - PUCSP - São Paulo (SP), Brasil; Docente do Curso de Especialização em Fonoaudiologia - VOZ da Pontifícia Universidade Católica de São Paulo - PUCSP - São Paulo (SP), Brasil.

(3) Doutora, Professora do Curso de Fonoaudiologia e do Programa de Estudos Pós Graduados em Distúrbios da Comunicação da Universidade Tuiuti do Paraná - UTP - Curitiba (PR), Brasil.

(4) Mestre, Professora do Curso de Especialização em Voz da Escola Superior da Amazônia - ESAMAZ - Belém (PA), Brasil; Coordenadora Administrativa do Curso de Especialização em Fonoaudiologia Hospitalar da Escola Superior da Amazônia - ESAMAZ - Belém (PA), Brasil.

(5) Doutor, Professor Titular da Universidade Tuiuti do Paraná - UTP - Curitiba (PR), Brasil.

Endereço para correspondência: Léslie Piccolotto Ferreira. R. Jesuíno Bandeira, 73, Vila Romana, São Paulo - SP, CEP: 05048-080. E-mail: lesliepf@pucsp.br

Recebido em: 3/3/2008; Aceito em: 12/6/2008 e do aperfeiçoamento do conhecimento. A necessidade de operar a crítica interna da produção tem sido apontada como condição para que o conhecimento produzido possa ser incorporado pela comunidade e cumprir o seu papel transformador ${ }^{(1-4)}$.

Estudos apontam para a crescente preocupação com o monitoramento da produção científica no plano internacional e no Brasil, razão pela qual, pesquisas vêm sendo desenvolvidas nacionalmente objetivando situar o Brasil na produção científica mundial $^{(1,5-5)}$.

Tais estudos evidenciam a necessidade de pesquisas que abordem questões como a dispersão - concentração da produção do conhecimento em relação aos veículos de divulgação, à temática, à temporalidade, à autoria, à regionalidade e à afiliação institucional a partir de análises que tenham como domínio de investigação a trajetória específica da área, bem como daquelas que estabeleçam o cotejamento de tal trajetória com a de áreas afins ${ }^{(1,5-6)}$.

A publicaçäo de resultados de pesquisas científicas tem sido o recurso mais utilizado para legitimar e registrar o avanço do conhecimento, sendo o periódico o meio mais utilizado para esse tipo de publicação ${ }^{(7-8)}$. Embora não haja uma correspondência absoluta entre produção científica e publicação, é fato internacionalmente reconhecido que, dentre os principais parâmetros para a mensuração do vigor científico de uma determinada área, encontram-se o volume de artigos publicados em periódicos indexados em bases de dados de prestígio e o 
número de citações que recebem, registrados nesses mesmos veículos $^{(1,7)}$. Considera-se, ainda, que se a produção científica é a atividade por excelência do pesquisador e uma das condições materiais para a socialização da atividade científica, tal produção deve ser publicada para efetivamente cumprir o seu papel $^{(9-11)}$. Estudos ressaltam que divulgação/publicação do conhecimento, embora consista na fase final de um trabalho, é tão importante como as etapas anteriores envolvidas no processo da pesquisa propriamente dita. Enfim, considera-se que a pesquisa não cumpre seu objetivo se não for visualizada pelos pares e disponibilizada à sociedade ${ }^{(4,11)}$.

Participando de forma decisiva da trajetória da produção bibliográfica acadêmica das diferentes áreas de conhecimento, encontram-se os periódicos ${ }^{(9,11-12)}$, uma vez que:

- se constituem em espaço aglutinador do conhecimento científico produzido;

- são veículos de maior visibilidade, uma vez indexados em bases de dados representativos nas respectivas áreas;

- se constituem no instrumento dinâmico e atual na veiculação e divulgação de tal conhecimento;

- participam da definição e da sistematização de critérios referentes aos aspectos estruturais, metodológicos e de mérito que norteiam o perfil de tal produção;

- se constituem como um dos instrumentos de avaliação e qualificação da produção bibliográfica científica produzida pela comunidade fonoaudiológica.

Destaca-se a importância de estudos que enfoquem a produção nos periódicos tendo em vista o papel fundamental que exercem na ampliação e consolidação da área em relação às suas atividades de formação e pesquisa. É urgente a necessidade do fortalecimento dos veículos já consolidados, bem como da criação de novos para que a área disponha e amplie os instrumentos representativos que garantam veiculação e o acesso do conhecimento produzido, fatores necessários para o estabelecimento do debate e da interlocução da comunidade científica. Enfim, é necessária a manutenção do fluxo e do ciclo de veiculação da produção de conhecimento para que a área avance o seu estágio de desenvolvimento ${ }^{(5,13)}$.

Estudos vêm sendo realizados no sentido de analisar o panorama da produção do conhecimento da área dos Distúrbios da Comunicação enfatizando a necessidade de reflexões em torno das temáticas e dos aspectos teórico-metodológicos que caracterizam a sua produção bibliográfica ${ }^{(4,7,11-12)}$.

Tais estudos são unânimes ao afirmar a importância da caracterização e análise dessa produção como condição para a área dos Distúrbios da Comunicação identificar os domínios de investigação que historicamente vem sendo priorizados e, a partir daí, estabelecer ações e campos estratégicos para o implemento da pesquisa ${ }^{(4,7,11-12)}$.

O objetivo desse estudo é analisar parte da produção de conhecimento voltada à área dos Distúrbios da Comunicação veiculada nos periódicos: Revista de Distúrbios da Comunicação (DIC); Revista da Sociedade Brasileira de Fonoaudiologia (RSBFa); Revista Fonoatual (Fono Atual) e Pró-Fono: revista de atualização científica (Pró-Fono), de circulação nacional, editados no período de 2000 a 2005. Os aspectos examinados foram: temática, faixa etária do público alvo, tipo de intervenção e contexto institucional.

\section{MÉTODOS}

\section{Material}

Foram objetos de análise deste estudo quatro periódicos especializados na área dos Distúrbios da Comunicação, publicados no Brasil, no período de 2000 a 2005: Distúrbios da Comunicação - DIC (início 1986); Pró-fono: revista de atualização científica - Pró-Fono (iniciada em 1989); Fonoatual - FA (início 1997); e Revista da Sociedade Brasileira de Fonoaudiologia - RSBF (início 1997). Adotou-se como critério de inclusão periódicos classificados no Qualis como maior ou igual à nacional B, em dezembro de 2005. O Qualis, concebido pela Coordenadoria de Aperfeiçoamento de Pessoal de Nível Superior (CAPES) ${ }^{(14)}$, é o resultado da classificação dos veículos utilizados pelos Programas de Pós Graduação para a divulgação de sua produção intelectual.

\section{Procedimento}

Para levantamento do material adotou-se: a identificação do volume, número e ano dos fascículos; a seleção dos artigos que compõe o corpus de análise; o mapeamento das características de cada artigo a partir da leitura do título, resumo e metodologia.

Optou-se como critério de inclusão:

- tipos de produção: artigo original, resenha, artigo de atualização, relato de caso, artigo de revisão.

Variáveis selecionadas:

- Temática: linguagem oral, linguagem escrita, voz, motricidade oral, saúde pública, disfagia, audiologia, otoneurologia, fundamentação educacional e profissional.

- Faixa etária do público alvo: de 0 a 3 meses, 4 meses a 3 anos, mais de 3 anos a 10 anos, mais de 10 anos a 16 anos, mais de 16 anos a 35 anos, mais de 35 anos a 65 anos e mais de 65 anos;

- Intervenção: triagem, prevenção, diagnóstico/avaliação, terapia individual, terapia em grupo, assessoria/promoção;

- Contexto institucional: clínica, escola, hospital, unidade básica de saúde (UBS), indústria/empresa, TV/rádio/teatro/ coral/orquestra/casa de espetáculo, e centro de reabilitação. Esse aspecto refere-se ao contexto abordado teoricamente e não necessariamente ao local de coleta de dados.

\section{Análise dos dados}

Todas as variáveis admitem ocorrências múltiplas. A classificação "sem identificação" foi adotada quando não foi possível caracterizar o artigo a partir das variáveis estabelecidas.

A análise estatística foi realizada por meio do Teste de Diferença de Proporções, ao nível de significância de 5\%.

\section{RESULTADOS}

Foram registrados 724 artigos (100 em 2000; 108 em 2001; 109 em 2002; 103 em 2003, 148 em 2004, 156 em 2005), em 71 fascículos (Figura 1). A Pró-Fono apresentou maior número de artigos 31,1\% (16 fascículos), a Fono Atual, 23,5\% (24 


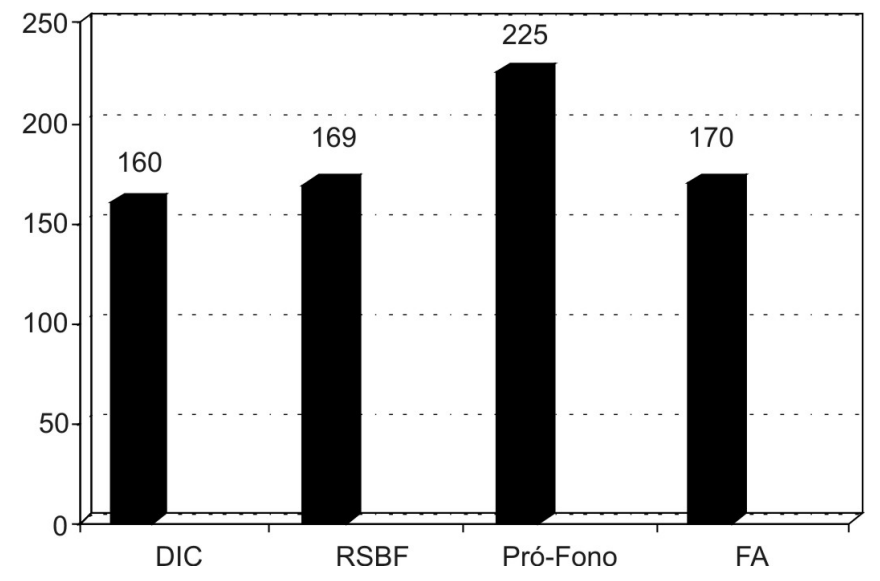

Legenda: $\mathrm{DIC}=$ Revista Distúrbios da Comunicação; RSBF = Revista da Sociedade Brasileira de Fonoaudiologia; FA = Revista Fono Atual

Figura 1. Número de artigos publicados (2000 a 2005)

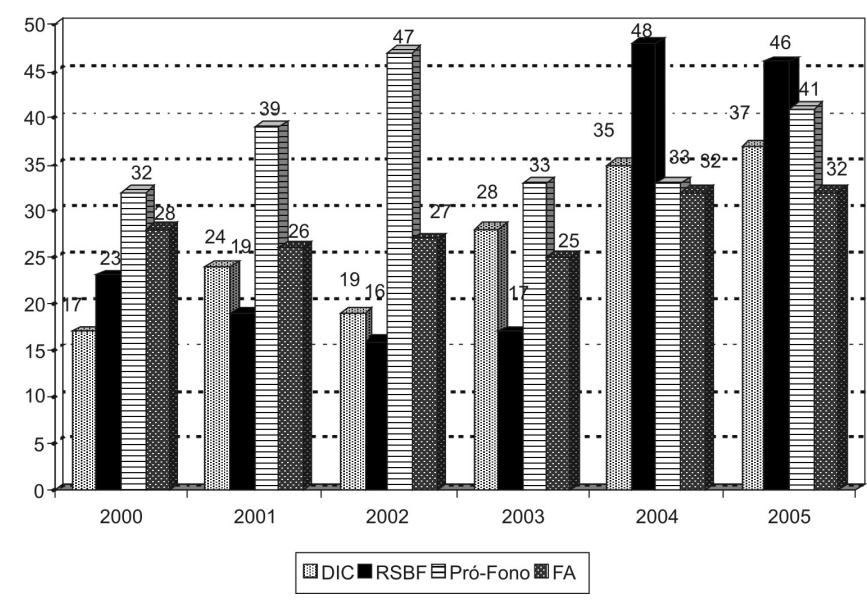

Legenda: DIC = Revista Distúrbios da Comunicação; RSBF = Revista da Sociedade Brasileira de Fonoaudiologia; FA = Revista Fono Atual

Figura 2. Comparação entre o número de artigos publicados nos periódicos (2000 a 2005)

fascículos), a RSBF, 23,3\% (16 fascículos) e a DIC, 22,1\% (15 fascículos) (Figura 2).

As Figuras 3, 4 e 5 apresentam ocorrências acerca da faixa-etária, do tipo de intervenção e contexto institucional, considerando a somatória das ocorrências isoladas e combinadas. Por exemplo, a temática linguagem oral foi abordada isoladamente 220 vezes e 63 de forma combinada com outras temáticas, daí o total de 283 ocorrências.

A temática mais pesquisada, isoladamente e de forma combinada, foi linguagem oral $(35,3 \%)$, seguida de audiologia $(28,9 \%)$, motricidade oral $(11,3 \%)$ e voz $(8,9 \%)$. As questões de linguagem escrita apareceram em poucas referências $(6,5 \%)$, seguidas por outras, como otoneurologia $(1,5 \%)$, disfagia $(2,0 \%)$ e saúde pública $(0,7 \%)$. Se a comparando as proporções das duas sub-áreas de maiores ocorrências (linguagem oral e audiologia), a diferença não é significativa ( $\mathrm{p}=0,0520)$, quando comparadas com as demais, elas apresentam produções significativamente maiores $(\mathrm{p}<0,001)$.

Quanto à faixa etária dos sujeitos envolvidos, isoladamente

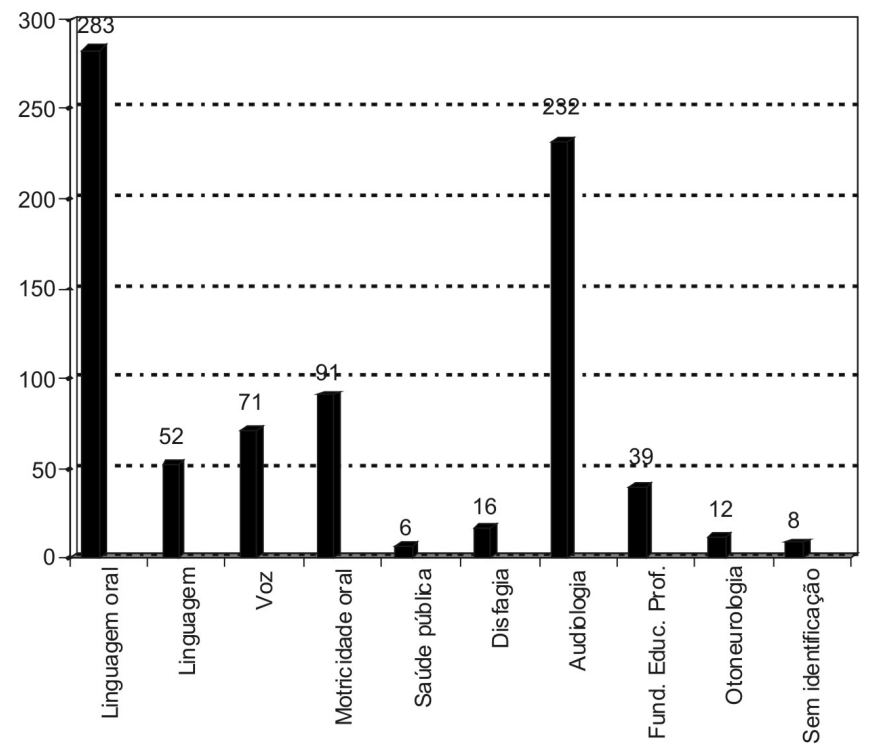

Legenda: Fund. Educ. Prof. = Fundamentação educacional e profissional

Figura 3. Distribuição das temáticas/sub-áreas isoladamente e de forma combinada

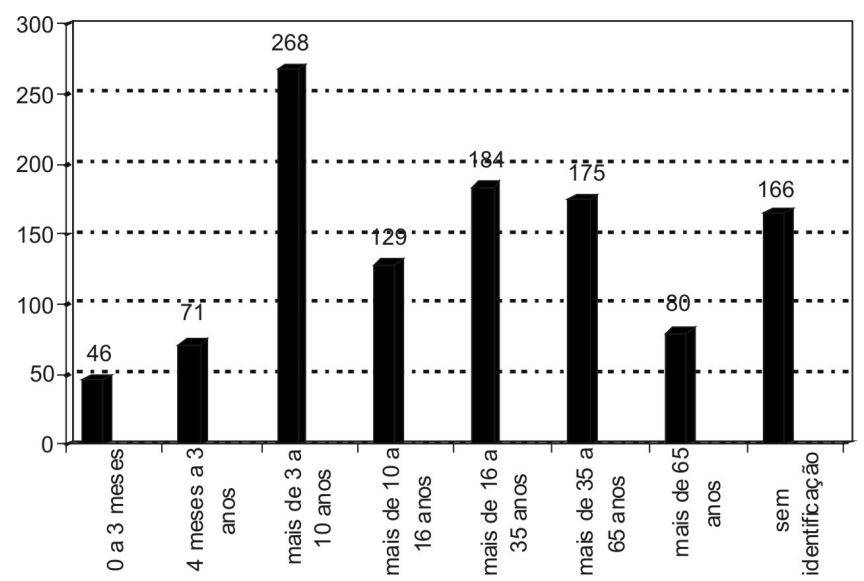

Figura 4. Distribuição das faixas etárias isoladamente e de forma combinada

e de forma combinada, verificou-se $22,8 \%$ com mais de 3 a 10 anos, $15,7 \%$ com mais de 16 a 35 anos e 14,9\% com mais de 35 a 65 anos. Menores registros foram constatados abordando os idosos $(6,8 \%)$ e bebês $(3,9 \%)$ e crianças de 4 meses a 3 anos $(6,1 \%)$. Comparando a proporção da faixa etária de maior ocorrência (mais de 3 a 10 anos) com as demais, observou-se que a proporção de artigos envolvendo a referida faixa etária é significativamente maior que as demais $(\mathrm{p}<0,0000)$.

A atuação fonoaudiológica de diagnosticar e/ou avaliar foi a mais referida de maneira isolada e combinada $(68,2 \%)$, seguida pela terapia individual $(15,8 \%)$. Comparando as proporções dessas intervenções, a diferença é significativa $(\mathrm{p}<0,001)$. A produção envolvendo diagnóstico/avaliação é significativamente maior que as demais.

Os artigos fazem maior referência ao contexto clínico (74,9\%), seguido do escolar $(8,7 \%)$ e hospitalar $(4,4 \%)$. Número restrito de pesquisas abordam atuações em indústria/ empresa (2,8\%) e TV/rádio/teatro/coral/orquestras/casas de es- 


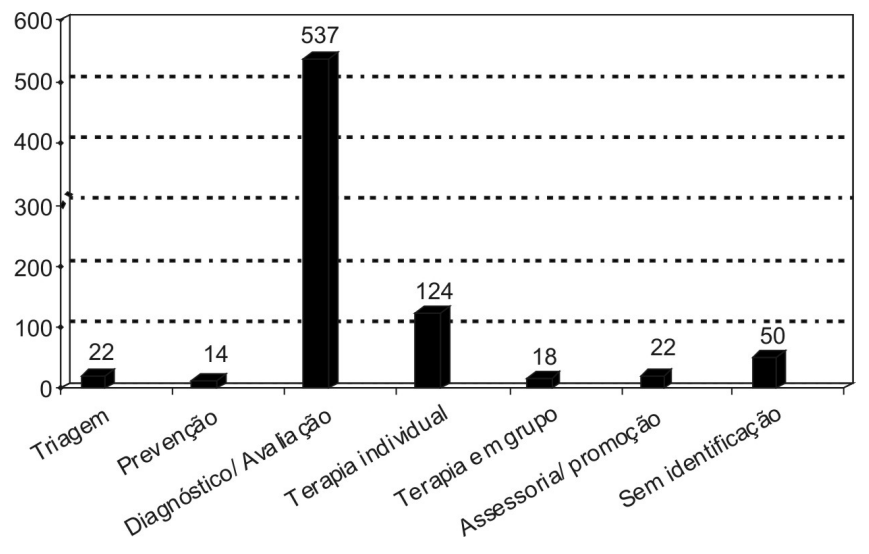

Figura 5. Distribuição dos tipos de intervenção isoladamente e de forma combinada

petáculo $(1,1 \%)$. Comparando os dois contextos institucionais de maiores ocorrências a diferença é significativa $(\mathrm{p}<0,001)$, ou seja, a produção de artigos envolvendo tais contextos é significativamente maior que os demais (Figura 6).

Os quadros a seguir apresentam dados referentes aos cruzamentos das faixas etárias, com os tipos de intervenção e temáticas. Nos Quadros 1, 2, 3 e 4 foram descritas, separadamente, as ocorrências com valor maior ou igual a 10. As ocorrências menores a esse valor foram agrupadas na categoria "outras". Por exemplo, a faixa etária de 0 a 3 meses, conforme a Figura

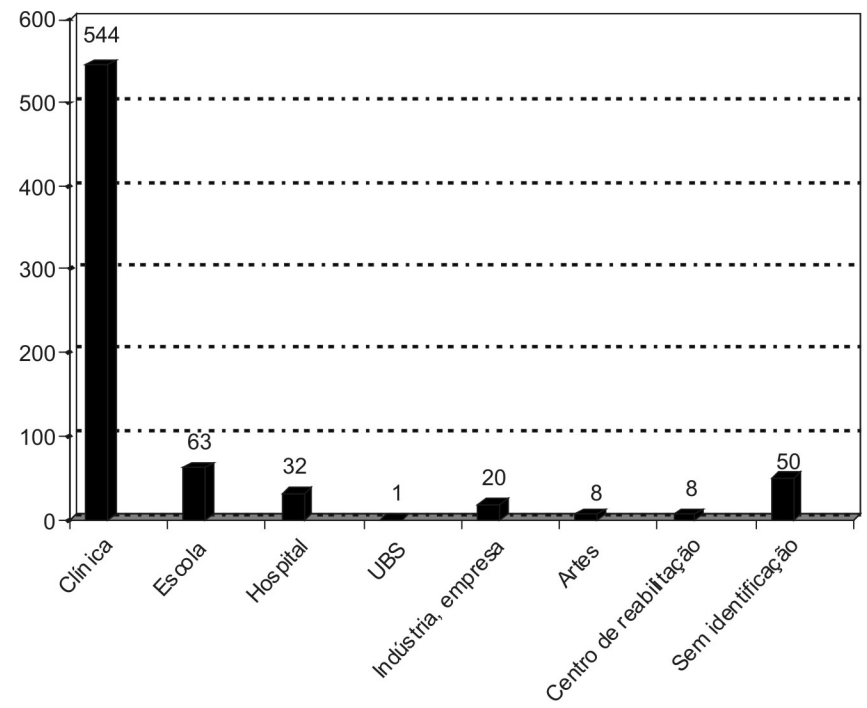

Legenda: UBS = Unidade Básica de Saúde; Artes =TV, rádio, teatro, coral, orquestras, casas de espetáculo

Figura 6. Distribuição dos contextos institucionais isoladamente e de forma combinada

4 aparece 46 vezes. Verifica-se no Quadro 1, 25 ocorrências isoladas e 13 de forma combinada com a faixa etária 4 meses a 3 anos. Sendo assim, 8 ocorrências referentes à faixa etária 0 a 3 meses, estão incluídas na categoria "outras".

Quadro 1. Distribuição percentual da relação entre as variáveis faixa etária e tipo de intervenção temáticas analisadas de forma isolada e combinada

\begin{tabular}{|c|c|c|c|c|c|c|c|c|c|c|}
\hline \multirow[t]{2}{*}{ Faixa etária } & \multicolumn{9}{|c|}{ Tipo de intervenção } & \multirow[t]{2}{*}{ Total (\%) } \\
\hline & $\mathrm{J}$ & $\mathrm{K}$ & L & $\mathrm{M}$ & $\mathrm{M}, \mathrm{N}$ & $\mathrm{N}$ & $\mathrm{P}$ & Q & Outras & \\
\hline A & 46 & 3 & 6 & 65 & 20 & 12 & 4 & 8 & 2 & $166(22,9)$ \\
\hline B & - & 4 & 1 & 18 & 1 & 1 & - & - & - & $25(3,4)$ \\
\hline $\mathrm{B}, \mathrm{C}$ & - & - & - & 7 & 2 & 3 & 1 & - & - & $13(1,8)$ \\
\hline C & - & - & - & 7 & 3 & 2 & - & - & - & $12(1,7)$ \\
\hline C,D & - & 1 & - & 24 & 2 & 1 & - & - & - & $28(3,9)$ \\
\hline D & 3 & 6 & 1 & 96 & 13 & 19 & 4 & 2 & 1 & $145(20)$ \\
\hline $\mathrm{D}, \mathrm{E}$ & - & 1 & 1 & 39 & 2 & 2 & 3 & 1 & 2 & $51(7)$ \\
\hline $\mathrm{D}, \mathrm{E}, \mathrm{F}$ & - & - & - & 7 & 1 & 2 & 1 & - & - & $11(1,5)$ \\
\hline E & - & 1 & - & 12 & 3 & 5 & 1 & 1 & - & $23(3,2)$ \\
\hline $\mathrm{F}$ & - & 1 & - & 28 & 2 & 6 & 1 & - & - & $38(5,3)$ \\
\hline$F, G$ & - & 2 & 2 & 57 & 1 & - & - & 2 & - & $64(8,8)$ \\
\hline$F, G, H$ & - & - & - & 25 & 2 & - & - & 2 & - & $29(4)$ \\
\hline G & 1 & - & - & 16 & 2 & 6 & - & 1 & - & $26(3,6)$ \\
\hline $\mathrm{G}, \mathrm{H}$ & - & 2 & - & 25 & 1 & 1 & - & - & - & $29(4)$ \\
\hline $\mathrm{H}$ & - & - & - & 8 & - & 2 & - & - & - & $10(1,4)$ \\
\hline Outras & - & 1 & - & 44 & 3 & 2 & 1 & 2 & 1 & $54(7,5)$ \\
\hline Total (\%) & $50(6,9)$ & $22(3)$ & $11(1,5)$ & $478(66)$ & $58(8)$ & $64(8,9)$ & $16(2,2)$ & $19(2,7)$ & $6(0,8)$ & $724(100)$ \\
\hline
\end{tabular}

Legenda: $A=$ sem identificação; $B=0$ a 3 meses; $C=4$ meses a 3 anos; $D=$ mais de 3 a 10 anos; $E=$ mais de 10 a 16 anos; $F=$ mais de 16 a 35 anos; $G=$ mais de 35 a 65 anos; $\mathrm{H}$ = mais de 65 anos; $\mathrm{J}=$ sem identificação; $\mathrm{K}=$ triagem; $\mathrm{L}$ = prevenção; $\mathrm{M}=$ diagnóstico/avaliação; $\mathrm{N}=$ terapia individual; $\mathrm{P}=$ terapia em grupo; $\mathrm{Q}$ = assessoria/promoção 
Quadro 2. Distribuição percentual da relação entre as variáveis faixas etárias e temáticas analisadas de forma isolada e combinada

\begin{tabular}{|c|c|c|c|c|c|c|c|c|c|c|c|c|}
\hline \multirow{2}{*}{$\begin{array}{l}\text { Faixa } \\
\text { etária }\end{array}$} & \multicolumn{11}{|c|}{ Temática } & \multirow[b]{2}{*}{ Total (\%) } \\
\hline & $\mathrm{K}$ & $\mathrm{K}, \mathrm{L}$ & $\mathrm{K}, \mathrm{N}$ & $\mathrm{K}, \mathrm{Q}$ & $\mathrm{L}$ & $\mathrm{M}$ & $\mathrm{N}$ & $\mathrm{Q}$ & $\mathrm{R}$ & $S$ & Outras & \\
\hline$A$ & 49 & 2 & 2 & 5 & 11 & 13 & 10 & 25 & 25 & - & 24 & $166(22,9)$ \\
\hline$B$ & 3 & - & 1 & 1 & - & - & 7 & 10 & 2 & - & 1 & $25(3,4)$ \\
\hline$B, C$ & 3 & - & 1 & 1 & - & - & 2 & 5 & - & - & 1 & $13(1,8)$ \\
\hline $\mathrm{C}$ & 3 & - & 1 & 1 & - & - & 3 & 3 & - & - & 1 & $12(1,7)$ \\
\hline C, D & 16 & - & 1 & 1 & - & 1 & 4 & 5 & - & - & - & $28(3,9)$ \\
\hline $\mathrm{D}$ & 77 & 8 & 2 & 10 & 13 & 2 & 11 & 16 & 3 & - & 3 & $145(20)$ \\
\hline $\mathrm{D}, \mathrm{E}$ & 21 & 1 & - & 3 & 1 & 2 & 7 & 15 & - & - & 1 & $51(7)$ \\
\hline $\mathrm{D}, \mathrm{E}, \mathrm{F}$ & 4 & - & - & - & - & - & - & 6 & 1 & - & - & $11(1,5)$ \\
\hline$E$ & 8 & 2 & - & 1 & 4 & 1 & 3 & 2 & - & - & 2 & $23(3,2)$ \\
\hline $\mathrm{F}$ & 9 & - & - & 1 & - & 6 & 4 & 16 & 1 & 1 & - & $38(5,3)$ \\
\hline $\mathrm{F}, \mathrm{G}$ & 6 & - & - & 2 & - & 14 & 4 & 29 & 1 & 6 & 2 & $64(8,8)$ \\
\hline $\mathrm{F}, \mathrm{G}, \mathrm{H}$ & 2 & - & 1 & - & - & 8 & - & 15 & - & 1 & 2 & $29(4)$ \\
\hline $\mathrm{G}$ & 3 & 1 & 1 & - & - & 6 & 1 & 11 & - & 1 & 2 & $26(3,6)$ \\
\hline $\mathrm{G}, \mathrm{H}$ & 3 & 1 & - & - & - & 3 & 5 & 9 & - & - & 8 & $29(4)$ \\
\hline $\mathrm{H}$ & 2 & - & - & - & 1 & 1 & 1 & 3 & - & 1 & 1 & $10(1,4)$ \\
\hline Outras & 11 & - & 2 & 3 & - & 6 & 7 & 22 & 1 & 2 & - & $54(7,5)$ \\
\hline Total (\%) & $220(30,4)$ & $15(2,1)$ & $12(1,7)$ & $29(4)$ & $30(4,1)$ & $63(8,7)$ & $69(9,5)$ & $192(26,5)$ & $34(4,6)$ & $12(1,7)$ & $48(6,6)$ & $724(100)$ \\
\hline
\end{tabular}

Legenda: $A=$ sem identificação; $B=0$ a 3 meses; $C=4$ meses a 3 anos; $D=$ mais de 3 a 10 anos; $E=$ mais de 10 a 16 anos; $F=$ mais de 16 a 35 anos; $G=$ mais de 35 a 65 anos; $\mathrm{H}=$ mais de 65 anos; $\mathrm{K}=$ linguagem oral; $\mathrm{L}=$ linguagem escrita; $\mathrm{M}=$ voz; $\mathrm{N}=$ motricidade oral; $\mathrm{P}=$ disfagia; $\mathrm{Q}=$ audiologia; $\mathrm{R}=$ fundamentação educacional e profissional; $\mathrm{S}=$ otoneurologia

Quadro 3. Distribuição percentual da relação entre as variáveis temáticas e tipos de intervenção temáticas analisadas de forma isolada e combinada

\begin{tabular}{|c|c|c|c|c|c|c|c|c|c|c|}
\hline \multirow[b]{2}{*}{ Temática } & \multicolumn{9}{|c|}{ Tipo de intervenção } & \multirow[b]{2}{*}{ Total (\%) } \\
\hline & $\mathrm{H}$ & $\mathrm{J}$ & $\mathrm{K}$ & $\mathrm{L}$ & $\mathrm{L}, \mathrm{M}$ & $M$ & $\mathrm{~N}$ & $\mathrm{P}$ & Outras & \\
\hline $\mathrm{A}$ & 9 & 7 & 4 & 129 & 29 & 28 & 10 & 3 & 1 & $220(30,4)$ \\
\hline$A, B$ & - & - & - & 6 & 4 & 4 & - & - & 1 & $15(2,1)$ \\
\hline$A, D$ & - & - & - & 9 & 2 & 1 & - & - & - & $12(1,7)$ \\
\hline$A, E$ & 2 & - & - & 16 & 3 & 4 & 1 & 2 & 1 & $29(4)$ \\
\hline $\mathrm{B}$ & 1 & 1 & - & 20 & 3 & 4 & - & 1 & - & $30(4,1)$ \\
\hline $\mathrm{C}$ & 2 & 2 & - & 41 & 2 & 6 & - & 9 & 1 & $63(8,7)$ \\
\hline $\mathrm{D}$ & 1 & 4 & 1 & 50 & 6 & 6 & 1 & - & - & $69(9,5)$ \\
\hline$E$ & 1 & 6 & 4 & 169 & 3 & 4 & 3 & 1 & 1 & $192(26,5)$ \\
\hline $\mathrm{F}$ & 23 & - & 1 & 5 & 2 & 1 & 1 & 1 & - & $34(4,6)$ \\
\hline $\mathrm{G}$ & - & - & - & 10 & - & 2 & - & - & - & $12(1,7)$ \\
\hline Outras & 11 & 2 & 1 & 23 & 4 & 4 & - & 2 & 1 & $48(6,6)$ \\
\hline Total (\%) & $50(6,9)$ & $22(3)$ & $11(1,5)$ & $478(66)$ & $58(8)$ & $64(8,9)$ & $16(2,2)$ & $19(2,7)$ & $6(0,8)$ & 724 (100) \\
\hline
\end{tabular}

Legenda: $A=$ linguagem oral; $B=$ linguagem escrita; $C=$ voz; $D=$ motricidade oral; $E=$ audiologia; $F=$ fundamentação educacional e profissional; $G=$ otoneurologia; $\mathrm{H}$ = sem identificação; $\mathrm{J}=$ triagem; $\mathrm{K}$ = prevenção; $\mathrm{L}$ = diagnóstico/avaliação; $\mathrm{M}=$ terapia individual; $\mathrm{N}$ = terapia em grupo; $\mathrm{P}=$ assessoria/promoção

Quadro 4. Distribuição percentual da relação entre as variáveis tipo de intervenção e contextos institucionais temáticas analisadas de forma isolada e combinada

\begin{tabular}{|l|c|c|c|c|c|c|c|}
\hline \multirow{2}{*}{ Tipo de intervenção } & \multicolumn{7}{|c|}{ Contexto institucional } \\
\cline { 2 - 8 } & $\mathrm{H}$ & $\mathrm{J}$ & $\mathrm{K}$ & $\mathrm{L}$ & $\mathrm{M}$ & Outras & Total (\%) \\
\hline A & 40 & 6 & 2 & - & - & 2 & $50(6,9)$ \\
\hline B & 2 & 9 & 7 & 2 & - & 2 & $22(3)$ \\
\hline C & 2 & 4 & 1 & 1 & 3 & - & $11(1,5)$ \\
\hline D & 4 & 390 & 39 & 22 & 15 & 8 & $478(66)$ \\
\hline D, E & - & 55 & - & 3 & - & - & $58(8)$ \\
\hline E & - & 60 & 2 & 1 & - & 1 & $64(8,9)$ \\
\hline F & - & 13 & - & 3 & - & - & $16(2,2)$ \\
\hline G & - & 2 & 11 & - & 2 & 4 & $19(2,7)$ \\
\hline Outras & 2 & 3 & 1 & - & - & - & $6(0,8)$ \\
\hline Total (\%) & $50(6,9)$ & $542(74,9)$ & $63(8,7)$ & $32(4,4)$ & $20(2,8)$ & $17(2,3)$ & $724(100)$ \\
\hline
\end{tabular}

Legenda: $A=$ sem identificação; $B=$ triagem; $C=$ prevenção; $D=$ diagnóstico/avaliação; $E=$ terapia individual; $F=$ terapia em grupo; $G=$ assessoria/promoção; $H=$ sem identificação; $\mathrm{J}=$ clínica; $\mathrm{K}$ = escola; $\mathrm{L}$ = hospital; $\mathrm{M}$ = indústria, empresa 


\section{DISCUSSÃO}

A partir desse estudo, de natureza cartográfica, pode-se acompanhar o fortalecimento da produção científica desses periódicos, culminando com uma tendência crescente do número de fascículos e de artigos publicados. Resultados semelhantes foram obtidos em pesquisa realizada a partir da análise de sete periódicos específicos da área dos Distúrbios da Comunicação ${ }^{(7)}$, que evidenciou um crescimento significativo de fascículos e artigos publicados nos últimos 30 anos. Se, conforme as autoras, a área, em 1986, publicou quatro fascículos com 15 artigos e em 2001, 18 fascículos com 185 artigos, os resultados aqui apresentados, que envolveram apenas quatro desses sete periódicos, evidenciam em 2005 a publicação de 14 fascículos e 156 artigos. Somado a esse dado, no período de 1986 a 2001, as autoras encontraram a média de 8,9 artigos por fascículo, e no período de 2000 a 2005, observou-se um aumento dessa média para 10,19.

Se tal crescimento deve ser exaltado, verifica-se que o número de periódicos e de artigos publicados na área dos Distúrbios da Comunicação é inferior a de outras áreas da saúde com maior tempo e tradição em publicações. Estudo que analisou periódicos da Psicologia ${ }^{(9)}$, sinalizou que em 2000 haviam 20 indexados em bases de dados. Resultados que remetem à mesma análise são apresentados em outra pesquisa ${ }^{(1)}$, que se propôs a avaliar seis periódicos em Psicologia. Nessa, embora os periódicos tenham sido editados anteriormente ao período proposto neste artigo, encontrou 749 publicações. Da mesma forma, a análise de três periódicos da Psiquiatria, entre 1981 a 1995, registrou $1474 \operatorname{artigos}^{(2)}$.

Problemas que afetam a produção nacional relativos à regularidade, à distribuição, à normalização, ao corpo editorial, ao sistema de arbitragem, ao impacto dos artigos relativizam esses números, e sinalizam para os aspectos que devem ser priorizados visando a consolidação das revistas nacional e internacionalmente $e^{(1,3,5-6,6,13)}$.

Quanto às temáticas, as mais pesquisadas, linguagem oral, seguida de audiologia, também foram observadas em pesquisa que analisou as 151 teses de doutorado de fonoaudiólogos brasileiros, desde a primeira defesa, em 1976, até abril de $2002^{(4)}$. Conforme o Quadro 3, a audiologia é abordada em artigos que tratam de todos os tipos de intervenção, enquanto a linguagem escrita, motricidade oral, voz e otoneurologia são objetos de análise em intervenções do tipo diagnóstico/ avaliação e terapia individual; e a linguagem oral, além dessas intervenções, também em terapia em grupo.

Quanto à faixa etária, embora a hipótese inicial tenha sido confirmada, ou seja, a publicação em maior número de artigos que abordam crianças de 3 a 10 anos, verifica-se a ampliação do envolvimento de sujeitos em diferentes ciclos de vida. É importante esclarecer que o Sistema Único de Saúde, que teve seus princípios estabelecidos na Lei Orgânica de Saúde em 1990 , tem contribuido para a mudança desse panorama ${ }^{(15)}$, ao considerar os princípios: da universalidade ("A saúde é um direito de todos") e de equidade ("todos devem ter igualdade de oportunidade em usar o sistema de saúde")(16). Quando a faixa etária é cruzada com as temáticas (Quadro 2), constatase que todas são pesquisadas e o predomínio de estudos sobre linguagem oral, envolve crianças de 4 meses a 10 anos, e da linguagem escrita, crianças de 3 a 10 anos. Enquanto a motricidade oral é mais pesquisada em crianças, adolescentes e adultos, há uma tendência dos artigos que abordam a voz priorizarem adultos.

O Quadro 1 evidencia que para todas as faixas etárias, o diagnóstico/avaliação aparece em maior número, a triagem e a terapia em grupo é mais pesquisada, em crianças até 10 anos e a terapia individual em crianças, mais do que em adultos, e a prevenção é pouco referida para todas as faixas etárias.

Chama atenção a maior recorrência para artigos que versavam apenas sobre o diagnosticar/avaliar. Tendo em vista, mais uma vez a implantação do Sistema Único de Saúde, verifica-se a necessidade de mudança nesse cenário, uma vez que o princípio da Integralidade prevê atenção à saúde, tanto por meios curativos, quanto preventivos; de forma individual ou coletiva ${ }^{(16)}$.

O Quadro 4 evidencia a tendência dos artigos versarem sobre diagnóstico e/ou avaliação, seguido de terapia individual, em contexto, predominantemente, clínico. Um número restrito de artigos refere-se à terapia em grupo ou assessoria e promoção de saúde.

\section{CONCLUSÃO}

A pesquisa evidencia a ampliação da produção do conhecimento nos periódicos abordados, com melhora gradual quanto ao nível e qualidade. Evidência também, a necessidade de uma distribuição mais equilibrada das temáticas, faixas etárias, tipos de intervenções e contextos institucionais abordados nos artigos veiculadas nos periódicos analisados. Novos levantamentos devem ser realizados, não apenas sob com o foco voltado para o registro histórico do que tem sido pesquisado, mas para subsidiar a definição de ações e campos estratégicos para o implemento da pesquisa na área dos Distúrbios da Comunicação. 


\begin{abstract}
Purpose: To analyze part of the knowledge production in the field of Speech-Language Pathology and Audiology. Methods: The articles published in the following journals from the year 2000 to 2005 were analyzed: Revista de Distúrbios da Comunicação; Revista da Sociedade Brasileira de Fonoaudiologia; Revista Fonoatual and Revista Pró-fono. The studied variables were: topic, age range of the studied population, type of intervention, and institutional context. Results: Seven hundred and twenty four articles were published during the period analyzed. The prevailing subject, either individually or in combination with other topics, was Oral Language (282), followed by Audiology (232), Orofacial Myology (91) and Voice (62). The prevailing age range studied was children from three to ten years old (145). Diagnosis and/or evaluation was the most mentioned type of intervention (547), in clinical (542), school (63) and hospital (32) contexts. Conclusion: Results reflected an increase of publications in the area during the studied period. The need for a more balanced distribution of topics, age groups, types of intervention, and institutional contexts in future publications is discussed. Further studies regarding knowledge production in the area would allow not only the historic registry of what has been investigated by speech-language therapists and audiologists, but also the definition of actions and strategic fields for implementing research in the area.
\end{abstract}

Keywords: Periodicals; Scientific and technical publications; Statistics \& numerical data; Research; Science

\section{REFERÊNCIAS}

1. Yamamoto OH, Menandro PRM, Koller SH, LoBianco AC, Hutz CS, Bueno JLO, Guedes MC. Avaliação de periódicos científicos brasileiros da área da psicologia. Ciênc Inf. 2002;31(2):163-77.

2. Figueira I, Leta J, Meis L. Avaliação da produção científica dos principais periódicos brasileiros de psiquiatria no período de 1981 a 1995. Rev Bras Psiquiatr. 1999;21(4):201-8.

3. Castro MLS. Educação e Economia: análise de artigos publicados em periódicos brasileiros: 1982-2000. Análise. 2006;17(2):224-33.

4. Russo ICP, Ferreira LP. Fonoaudiólogos doutores no Brasil: análise das teses segundo áreas de atuação e programas. Pró-Fono. 2004;16(1):11930 .

5. Strehl L. O fator de impacto do ISI e a avaliação da produção científica: aspectos conceituais e metodológicos. Ciênc Inf. 2005;34(1):19-27.

6. Trzesniak P. As dimensões da qualidade dos periódicos científicos e sua presença em um instrumento da área da educação. Rev Bras Educ. 2006;11(32):346-61.

7. Campanatti-Ostiz H, Andrade CRF. Periódicos nacionais em Fonoaudiologia: caracterização estrutural. Rev Soc Bras Fonoaudiol. 2005;10(3):147-54.

8. Bufrem LS, Sorribas TV. Produção científica em Ciência da Informação: análise temática em artigos de revistas brasileiras. Perspect Ciênc Inf. 2007;12(1):38-49.
9. Sampaio MIC, Peixoto ML. Periódicos brasileiros de psicologia indexados nas bases de dados Lilacs e PsycINFO. Bol Psicol. 2000;50(112):65-73.

10. Ferreira NS, Yoshida EMP. Produção científica sobre psicoterapias breves no Brasil e demais países latino-americanos (1990-2000). Estud Psicol (Natal). 2004;9(3):523-31.

11. Campanatti-Ostiz H, Andrade CRF. Periódicos nacionais em Fonoaudiologia: caracterização de indicador de impacto. Pró-Fono. 2006;18(1):99-110.

12. Campanatti-Ostiz H, Andrade CRF, Barbosa MA. Considerações teóricas sobre a escolha de descritores na área Fonoaudiológica. PróFono. 2003;15(2):211-8.

13. Rebelatto JR, Albuquerque F. Delineamento de linha de base na investigacao científica em fisioterapia. Rev Bras Fisioter. 2004;8(1):6774.

14. Coordenadoria de Aperfeiçoamento de Pessoal de Nível Superior (Capes).Classificação de periódicos, anais, revistas e jornais [Internet]. Brasília (DF):Capes; c2008. [citado 2009 Jan 22]. Disponível em: http:// qualis.capes.gov.br/

15. Prado SD, Sayd JD. A produção científica sobre envelhecimento e saúde no Brasil. Textos Envelhecimento. 2004;7(2):85-101.

16. Ministério da Saúde [Internet]. Brasil. Sobre o SUS [cited 2007 Dec 29] Available from: http://portal.saude.gov.br/portal/saude. 